\title{
铂钴合金纳米电催化剂的制备及性能研究
}

$$
\text { 朱 勇 }{ }^{1} \text {, 顾 军 }{ }^{1,2} \text {, 于 } \text { 涛 }^{1,3} \text {, 何海佟 }{ }^{1} \text {, 姚 } \text { 嗆 }^{1}
$$

(1. 南京大学 物理学院, 南京 210093; 2. 南京东炎氢能源科技有限公司, 南京 $211100 ; 3$. 南京大学 固体微结构 物理国家重点实验室，南京 210093)

摘 要: 研制高活性的电催化剂是实现质子交换膜燃料电池的商业化应用必须解决的关键技术之一。本研究以三乙 胺为碱性络合剂、硼氢化钠为还原剂, 采用液相合成法制备 PtCo 纳米合金电催化剂, 再通过高温热处理实现最佳 电化学性能。采用各种表征方法对催化剂的微观结构及电化学性能进行测定, 探究硼氢化钠、三乙胺的添加量及高 温热处理对催化剂电化学性能的影响。结果显示, 适量的硼氢化钠可提升催化剂的电化学活性面积, 三乙胺可以改 变催化剂的质量活性, 高温热处理能有效提升催化剂的质量活性, 极大提升催化剂的氧还原反应(ORR)能力; 在同 一测试体系下, 添加 $100 \mathrm{mg}$ 硼氢化钠及 $100 \mu \mathrm{L}$ 三乙胺在 $500{ }^{\circ} \mathrm{C}$ 高温热处理条件下制备的 PtCo 纳米合金电催化剂 的质量活性达到 $133 \mathrm{~mA} / \mathrm{mg}_{\mathrm{Pt}}$, 是田中贵金属工业株式会社(TKK)商用 PtCo 合金催化剂的 3 倍。

关 键 词: 电催化剂; PtCo 合金; 高温热处理; 氧还原反应; 电化学活性面积

中图分类号: 0646 文献标识码: A

\section{Synthesis and Property of Platinum-cobalt Alloy Nano Catalyst}

$$
\text { ZHU Yong }^{1} \text {, GU Jun }{ }^{1,2} \text {, YU Tao }{ }^{1,3} \text {, HE Haitong }{ }^{1} \text {, YAO Rui }{ }^{1}
$$

(1. College of Physics, Nanjing University, Nanjing 210093, China; 2. Nanjing Doinpower Technology Co., Ltd., Nanjing 211135, China; 3. National Laboratory of Solid-State Microstructures, Nanjing University, Nanjing 210093, China)

\begin{abstract}
In order to achieve the commercialization of proton exchange membrane fuel cells (PEMFCs), it is necessary to synthesize electrocatalyst with higher electrochemical activity. In this study, PtCo nano-alloy electrocatalyst was prepared by liquid phase synthesis method with sodium borohydride as reducing agent, triethylamine as complexing agent, and by sequential heat-treatment. The physical properties of the catalyst were characterized by different analytical methods. We studied the effects of heat-treatment temperature, different amounts of sodium borohydride and triethylamine on electrochemical performance. The results show that heat-treatment can greatly improve the mass activity of the catalyst, and $500{ }^{\circ} \mathrm{C}$ is the optimal temperature for preparing the catalyst with the highest catalytic performance towards oxygen reduction reaction(ORR). Compared with commercial TKK-PtCo alloy catalyst under the same test system, the as-prepared catalysts exhibits advantages of more uniform particle size distribution, smaller particle size and higher electrochemical performance. In particular, the mass activity (MA) of the prepared catalyst is $133 \mathrm{~mA} / \mathrm{mg}_{\mathrm{Pt}}$, which is 3 times of TKK-PtCo alloy catalyst.
\end{abstract}

Key words: electrocatalyst; platinum-cobalt alloy; heat-treatment; oxygen reduction reaction; active specific surface area

收稿日期: 2020-05-12；收到修改稿日期：2020-07-06; 网络出版日期：2020-08-01

基金项目: 中央高校基本科研业务费专项资金(14380168) Fundamental Research Funds for the Central Universities (14380168)

作者简介: 朱 勇(1994-), 男, 硕士研究生. E-mail: Zyong_mail@yeah.net ZHU Yong(1994-), male, Master candidate. E-mail: Zyong_mail@yeah.net

通信作者：顾 军，副教授. E-mail: junguca@nju.edu.cn GU Jun, associate professor. E-mail: junguca@nju.edu.cn 
随着化石能源日趋突出的供求矛盾, 以及因化 石能源不安全性、环境污染弊端带来的负面影响日 益深化, 探究可持续能源转换电池技术的需求变得 十分迫切 ${ }^{[1]}$ 。作为能够替代化石燃料设备的燃料电 池具有广阔的运用前景。燃料电池中, 质子交换膜 燃料电池(PEMFCs) 是最有希望替代内燃机作为交 通动力的设备之一, 具有无污染、燃料来源丰富、 便携、高效等特点 ${ }^{[2]}$ 。为实现质子交换膜燃料电池 的商业化，制备高效耐用的电催化材料必不可少。 因此, 燃料电池中电化学反应机理及其相关动力学 参数研究成为电催化领域的热点研究方向 ${ }^{[3]}$ 。

碳支撑的金属纳米材料是燃料电池常用的电催 化剂 ${ }^{[4]}$ 。其中, $\mathrm{Pt}$ 具有理想的电催化表面, 可以选择 性地激活分子氧的结合, 具有良好的氧还原能力 ${ }^{[5]}$, 可作为氧还原反应 $(O R R)$ 和氢氧化反应 $(H O R)$ 的主 要催化剂 ${ }^{[6]}$ 。但由于氧还原反应是缓慢的动力学过 程, 且 Pt 纳米粒子固有的不稳定性, 在燃料电池的 工作过程中 $\mathrm{Pt}$ 基电催化剂的 $\mathrm{ORR}$ 能力受限 ${ }^{[7]}$ 。因 此, 研制更高活性的电催化剂是加快 PEMFCs 应用 必须解决的关键技术之一。

近年来, 为提高电催化剂的 ORR 活性和稳定性, 已开展对电催化剂载体的改进、金属合金材料的合 成等研究工作 ${ }^{[8-9]}$ 。已有研究表明, Pt-M 基( $M=M n$, $\mathrm{Fe}, \mathrm{Co}, \mathrm{Ni}, \mathrm{Cu}, \mathrm{Zn}$ )双金属和三金属电催化剂可提高 ORR 能力和稳定性 ${ }^{[10]}$, 原因在于合金表面 $\mathrm{d}$ 带中心 偏移, Pt 的电子结构因过渡金属而改变, 使得氧原 子结合能降低, ORR 能力提高 ${ }^{[11]}$ 。在过渡金属中, 从 催化剂活性、金属间化合物的形成难易度的观点来 看 ${ }^{[12]}, \mathrm{Co}$ 更容易与 $\mathrm{Pt}$ 形成金属间化合物 ${ }^{[13]}$ 。 $\mathrm{Li}^{[14]}$ 和 $\mathrm{Bai}$ 等 ${ }^{[15]}$ 研究表明, 引入 $\mathrm{Co}$ 可有效改善 $\mathrm{Pt}$ 基电 催化剂的 ORR 性能。

本研究使用常用的工业原料合成具有良好电化 学活性面积 (ECSA) 和氧还原反应 (ORR) 能力的 $\mathrm{PtCo}$ 合金纳米电催化剂; 探究引入 $\mathrm{Co}$ 后, 合成 $\mathrm{PtCo}$ 合金纳米电催化剂所需还原剂 $\left(\mathrm{NaBH}_{4}\right)$ 和碱性 络合剂(三乙胺)的最佳添加量及高温热处理的最佳 温度。

\section{1 实验方法}

\section{1 典型铂钴合金纳米电催化剂的合成}

取 $1.504 \mathrm{~g}$ 氯铂酸 $\left(\mathrm{H}_{2} \mathrm{PtCl}_{6} \cdot 6 \mathrm{H}_{2} \mathrm{O}\right.$, 昆明铂锐金 属材料有限公司)水溶液 ( $10 \mathrm{wt} \%$ ) 和 $21.78 \mathrm{mg}$ 氯化钴 $\left(\mathrm{CoCl}_{2} \cdot 6 \mathrm{H}_{2} \mathrm{O}, \geqslant 99.8 \%\right.$, 国药集团化学试剂有限公 司)加入 $25 \mathrm{~mL}$ 无水乙醇 $(\leqslant 0.005 \%$ 水, 国药集团化
学试剂有限公司)中, 超声 $10 \min (60 \mathrm{~W}$, 无锡上佳 生物科技有限公司)混合均匀。取 $100 \mu \mathrm{L}$ 三乙胺 $\left(\left(\mathrm{CH}_{2} \mathrm{CH}_{3}\right)_{3} \mathrm{~N}, \geqslant 99.9 \%\right.$, 国药集团化学试剂有限公 司)滴入混合液中, 超声至浑浊均匀状态, 得到前 驱液。在冰浴条件下, 取 $57.84 \mathrm{mg}$ 碳黑(EC-600JD, 日本狮王株式会社)加入前驱液中, 超声 $1 \mathrm{~h}$ 得混合 浆料。

混合浆料在磁力搅拌 (1000 r/min) 条件下, 加入 $100 \mathrm{mg} \mathrm{NaBH} 4$ ( $\geqslant 96.0 \%$, 国药集团化学试剂有限公 司), 搅拌 $1 \mathrm{~h}$ 后, 超纯水洗涤 3 次, 在 $80{ }^{\circ} \mathrm{C}$ 真空干 燥 $10 \mathrm{~h}$, 得到典型 $\mathrm{PtCo}$ 合金纳米电催化剂, 标记为 $\mathrm{Pt}_{2.8} \mathrm{Co} / \mathrm{C}$ 。继续对 $\mathrm{Pt}_{2.8} \mathrm{Co} / \mathrm{C}$ 进行高温热处理: 升温 梯度 $8.3{ }^{\circ} \mathrm{C} / \mathrm{min}$, 在 $\mathrm{H}_{2}$ 浓度为 $20 \%$ 的 $\mathrm{H}_{2} / \mathrm{N}_{2}$ 混合气 条件下, 不同高温处理 $2 \mathrm{~h}$ 。将高温热处理的 $\mathrm{Pt}_{2.8} \mathrm{Co} / \mathrm{C}$ 标记为 $\mathrm{Pt}_{2.8} \mathrm{Co} / \mathrm{C}-T$, 其中 $T$ 为具体温度。

根据 $\mathrm{Pt}$ 与 $\mathrm{Co}$ 的添加比例, 其它条件不变, 未高 温热处理且只改变嗍氢化钠添加量所制备的催化剂

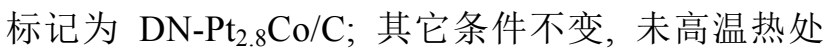
理且只改变三乙胺添加量所制备的催化剂标记为 DTL-Pt $2.8 \mathrm{Co} / \mathrm{C}$; 将进行加速稳定性测试后的催化剂 样品标记为 ADT-M(M 为本文中所命名的催化剂)。 此外, TKK 商用 PtCo 合金催化剂(TEC36E52, 金属 载量 $52 \mathrm{wt} \%$, 其中 $\mathrm{Pt}$ 为 $47 \mathrm{wt} \%$, Co 为 $5 \mathrm{wt} \%$ ) 标记为 $\mathrm{TKK}-\mathrm{PtCo} / \mathrm{C}$ 。本研究中所制备的电催化剂金属载量 均与 $\mathrm{TKK}-\mathrm{PtCo} / \mathrm{C}$ 保持一致。

\section{2 催化剂物理表征}

使用透射电子显微镜(TEM, TECNAI F20)、能 量色散 X 射线光谱(EDS)、 $X$ 射线衍射(XRD, Bruker D8 Adavance)对 PtCo 合金纳米电催化剂的晶型结 构、形貌特征和物理特性进行表征。

\section{3 电化学性能测试}

用 $\mathrm{Al}_{2} \mathrm{O}_{3}$ 粉末 $(0.05 \mu \mathrm{m})$ 将玻璃碳电极 $(d=5 \mathrm{~mm})$ 表面打磨光滑。取 $2 \mathrm{mg}$ 样品, 加入 $600 \mu \mathrm{L}$ 超纯水, 超声 $3 \mathrm{~min}$ 后, 加入 $200 \mu \mathrm{L}$ 异丙醇 $(\geqslant 99.9 \%$, 国药 集团化学试剂有限公司)。在冰浴条件下, 超声 $10 \mathrm{~min}$ 。取 $50 \mu \mathrm{L}$ Nafion( $5 \mathrm{wt} \%$ ，美国 Cobot 公司)滴 入混合液中, 超声 $30 \mathrm{~min}$ 后取 $10 \mu \mathrm{L}$ 混合浆料滴至 玻璃碳电极表面, 均匀成膜 ${ }^{[16]}$ 。

干燥成膜后, 采用 Gamry 公司的 Reference 3000 电化学工作站进行电化学测试和相关数据记 录。采用标准三电极体系测试循环伏安曲线 $(\mathrm{CV})$ 以 计算 ECSA, 测试线性伏安曲线( LSV)以计算质量活 性 (MA), 参比电极为饱和甘录电极 ( $\mathrm{SCE}$ ), 铂丝 $(d=0.5 \mathrm{~mm})$ 为对电极, 工作电极为玻璃碳电极 $(d=$ $5 \mathrm{~mm}, S=0.196 \mathrm{~cm}^{2}$ ), 电解液为 $0.1 \mathrm{~mol} / \mathrm{L}$ 的 $\mathrm{HCLO}_{4}$ 
水溶液。

在 $0.1 \mathrm{~mol} / \mathrm{L}$ 的氮气饱和 $\mathrm{HCLO}_{4}$ 溶液中, 进行 循环伏安 $(\mathrm{CV})$ 测试。活化过程: 扫描电位 0.05 $1.2 \mathrm{~V}(v s \mathrm{RHE})$, 扫速 $200 \mathrm{mV} / \mathrm{s}$, 扫描 20 圈。CV 测 试过程: 扫描电位 0.05 1.2 V(vs RHE), 扫速 $20 \mathrm{mV} / \mathrm{s}$, 扫描 3 圈。完成 $\mathrm{CV}$ 测试后, 在 $0.1 \mathrm{~mol} / \mathrm{L}$ 的氧气饱 和 $\mathrm{HCLO}_{4}$ 溶液中, 进行线性伏安曲线( $\mathrm{LSV}$ )测试, 扫描电位为 $0.05 \sim 1.05 \mathrm{~V}$ (vs RHE), 扫速 $10 \mathrm{mV} / \mathrm{s}$, 电 极旋转速度为 $1600 \mathrm{r} / \mathrm{min}$ 。加速稳定性测试(ADT): $0.1 \mathrm{~mol} / \mathrm{L}$ 的氮气饱和 $\mathrm{HCLO}_{4}$ 溶液条件下, 扫描电位 为 $0.6 \sim 1.2 \mathrm{~V}(v s \mathrm{RHE})$, 扫速 $100 \mathrm{mV} / \mathrm{s}$, 扫描 5000 圈。

ECSA 和 MA 的计算方法如下:

$$
\begin{gathered}
\mathrm{ECSA}=\left(\frac{Q(\mathrm{C})}{Q(\mathrm{AC})}\right) \times \frac{1}{L_{\mathrm{Pt}}} \\
\mathrm{MA}=\frac{i_{k}}{m_{\mathrm{Pt}}}
\end{gathered}
$$

其中, $Q(\mathrm{C})\left(\mathrm{mC} \cdot \mathrm{cm}^{-2}\right)$ 是氢脱吸附区域的库仑电荷; $L_{\mathrm{Pt}}\left(\mathrm{mg} \cdot \mathrm{cm}^{-2}\right)$ 是玻璃碳电极上的 $\mathrm{Pt}$ 负载质量; $Q(\mathrm{AC})=$ $0.21 \mathrm{mC} \cdot \mathrm{cm}^{-2}$, 表示铂表面单层原子吸附氢的电荷 量; $i_{\mathrm{k}}$ 是由 $\mathrm{K}-\mathrm{L}$ 方程得出的动力学电流; $m_{\mathrm{Pt}}$ 是玻璃碳 电极上的铂载量。

\section{2 结果与讨论}

\section{1 物理性能表征}

图 1 为 $\mathrm{Pt}_{2.8} \mathrm{Co} / \mathrm{C} 、 \mathrm{Pt}_{2.8} \mathrm{Co} / \mathrm{C}-500$ 和 $\mathrm{TKK}-\mathrm{PtCo} / \mathrm{C}$ 的 XRD 图谱。样品都具有 $\mathrm{Pt}$ 和 $\mathrm{PtCo}$ 对应的典型面 心立方结构(fcc); $\mathrm{Pt}_{2.8} \mathrm{Co} / \mathrm{C}$ 与 $\mathrm{Pt}_{2.8} \mathrm{Co} / \mathrm{C}-500$ 相应衍 射峰的 $2 \theta$ 角比标准 $\mathrm{Pt}$ 单晶更大, 与 $\mathrm{TKK}-\mathrm{PtCo} / \mathrm{C}$ 衍 射峰相似且都向右偏移, 证明原子尺寸较小的 Co 进入了尺寸较大的 $\mathrm{Pt}$ 晶格 ${ }^{[17]}$ 。经过 $500{ }^{\circ} \mathrm{C}$ 高温热 处理后的催化剂, 衍射峰明显增强, 这说明催化剂 的结晶度更优; 此外, 衍射峰半高宽度缩小, 也说明

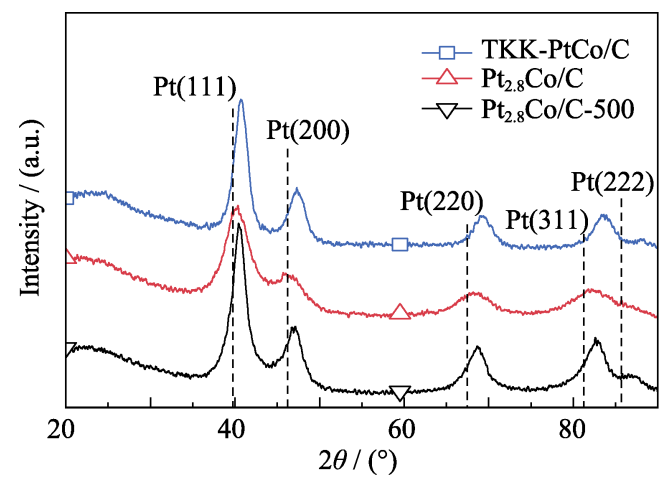

图 $1 \mathrm{Pt}_{2.8} \mathrm{Co} / \mathrm{C} 、 \mathrm{Pt}_{2.8} \mathrm{Co} / \mathrm{C}-500$ 和 TKK-PtCo/C 的 XRD 图谱 Fig. 1 XRD patterns of $\mathrm{Pt}_{2.8} \mathrm{Co} / \mathrm{C}, \mathrm{Pt}_{2.8} \mathrm{Co} / \mathrm{C}-500$ and $\mathrm{TKK}-\mathrm{PtCo} / \mathrm{C}$
催化剂尺寸增大。结合 XRD 图谱与 Bragg 公式计算 得到, $\mathrm{Pt}_{2.8} \mathrm{Co} / \mathrm{C} 、 \mathrm{Pt}_{2.8} \mathrm{Co} / \mathrm{C}-500$ 和 $\mathrm{TKK}-\mathrm{PtCo} / \mathrm{C}$ 的 $\operatorname{Pt}(111)$ 晶格间距分别为 $0.224 、 0.223$ 和 $0.221 \mathrm{~nm}$, 与 标准单晶 $\operatorname{Pt}(111)$ 的 $0.226 \mathrm{~nm}$ 相比均明显减小, 这与 XRD 衍射峰向右偏移的现象相吻合, 表明 $\mathrm{Pt}$ 和 Co 之间具有良好的合金化程度, 本工艺成功制备了 $\mathrm{PtCo}$ 合金催化剂。

图 2(a c) 分别为 $\mathrm{Pt}_{2.8} \mathrm{Co} / \mathrm{C} 、 \mathrm{Pt}_{2.8} \mathrm{Co} / \mathrm{C}-500$ 、 $\mathrm{TKK}-\mathrm{PtCo} / \mathrm{C}$ 的 TEM 照片及其粒径分布图。TEM 照 片显示的晶格间距与 XRD 计算结果近似; 其中, $\mathrm{Pt}_{2.8} \mathrm{Co} / \mathrm{C}-500$ 的晶格间距较 $\mathrm{Pt}_{2.8} \mathrm{Co} / \mathrm{C}$ 更小, 说明高 温热处理进一步提升了铂钴的合金化程度。三种催 化剂的形貌近似球形, $\mathrm{Pt}_{2.8} \mathrm{Co} / \mathrm{C}$ 与 $\mathrm{Pt}_{2.8} \mathrm{Co} / \mathrm{C}-500$ 较 为均匀地分布在碳载体上, 且颗粒尺寸差异较小, 但 $\mathrm{TKK}-\mathrm{PtCo} / \mathrm{C}$ 的颗粒差异较大。通过随机统计 100 个金属颗粒得到 $\mathrm{Pt}_{2.8} \mathrm{Co} / \mathrm{C} 、 \mathrm{Pt}_{2.8} \mathrm{Co} / \mathrm{C}-500$ 的平均颗 粒尺寸分别为 $3.45 、 3.85 \mathrm{~nm}$, 均小于 $\mathrm{TKK}-\mathrm{PtCo} / \mathrm{C}$ $(5.75 \mathrm{~nm})$ 。纳米金属颗粒更小, 才能更有效地提高 $\mathrm{Pt}$ 的利用率, 提供更多活性位点, 进而提升催化剂 的电催化能力 ${ }^{[3,18-20]}$ 。

图 3 为 $\mathrm{Pt}_{2.8} \mathrm{Co} / \mathrm{C}-500$ 的能量色散 $\mathrm{X}$ 射线光谱图 (EDS)。由图可见, Co 与 $\mathrm{Pt}$ 元素分布较为均一, 且 元素分布区域与选定区域相同, 进一步说明成功合 成了 $\mathrm{PtCo}$ 合金纳米电催化剂。此外, 图 $\mathrm{S} 1$ 为 $\mathrm{Pt}_{2.8} \mathrm{Co} / \mathrm{C}-500$ 的能谱图, 可观察到金属 $\mathrm{Pt}$ 的峰强度 明显超过金属 Co, 峰面积也更大, 证明催化剂中 Pt 比 Co 的相对含量更高, 这与图 3(c, d) 的元素分布情 况一致。

\section{2 电化学性能表征}

图 4(a)为 $\mathrm{NaBH}_{4}$ 的添加量对于 $\mathrm{DN}-\mathrm{Pt}_{2.8} \mathrm{Co} / \mathrm{C}$ 的 ECSA 的影响。当 $\mathrm{NaBH}_{4}$ 的添加量控制在 $100 \mathrm{mg}$ 时, $\mathrm{Pt}_{2.8} \mathrm{Co} / \mathrm{C}$ 催化剂的 ECSA 达到最大值 $51.6 \mathrm{~m}^{2} / \mathrm{g}_{\mathrm{Pt}}$, 与 $\mathrm{TKK}$ 商用催化剂 $\left(28.1 \mathrm{~m}^{2} / \mathrm{g}_{\mathrm{Pt}}\right)$ 相比明显提高。这 是因为 $\mathrm{NaBH}_{4}$ 添加量会影响 $\mathrm{Pt}$ 晶核的生长, 从而改 变催化剂的 ECSA。适量的 $\mathrm{NaBH}_{4}$ 可以有效减少由 $\mathrm{PtCl}_{6}{ }^{2-}$ 阴离子团还原的 $\mathrm{Pt}$ 晶核间的团聚, 阻碍其它 $\mathrm{PtCl}_{6}{ }^{2-}$ 在 $\mathrm{Pt}$ 晶核上的吸附，从而限制晶核的继续生 长, 避免催化剂的颗粒尺寸过大。

由 $\mathrm{CV}$ 曲线(图 S2(a)) 可知, $\mathrm{NaBH}_{4}$ 添加量分别为 90、 $100 \mathrm{mg}$ 时所制备的 $\mathrm{DN}-\mathrm{Pt}_{2.8} \mathrm{Co} / \mathrm{C}$ 氢脱附区域面 积都较大, 说明其脱吸附氢原子的能力强于 $\mathrm{TKK}-\mathrm{PtCo} / \mathrm{C}^{[21]}$ 。由图 S2(b) 中 $\mathrm{LSV}$ 曲线可知, $\mathrm{DN}-\mathrm{Pt}_{2.8} \mathrm{Co} / \mathrm{C}$ 催化剂的半波电位均低于 $\mathrm{TKK}-\mathrm{PtCo} / \mathrm{C}$ 。

通过对 $\mathrm{NaBH}_{4}$ 的调控所制备催化剂的 ECSA 虽得到提升, 但合成的催化剂并未表现出较强的 

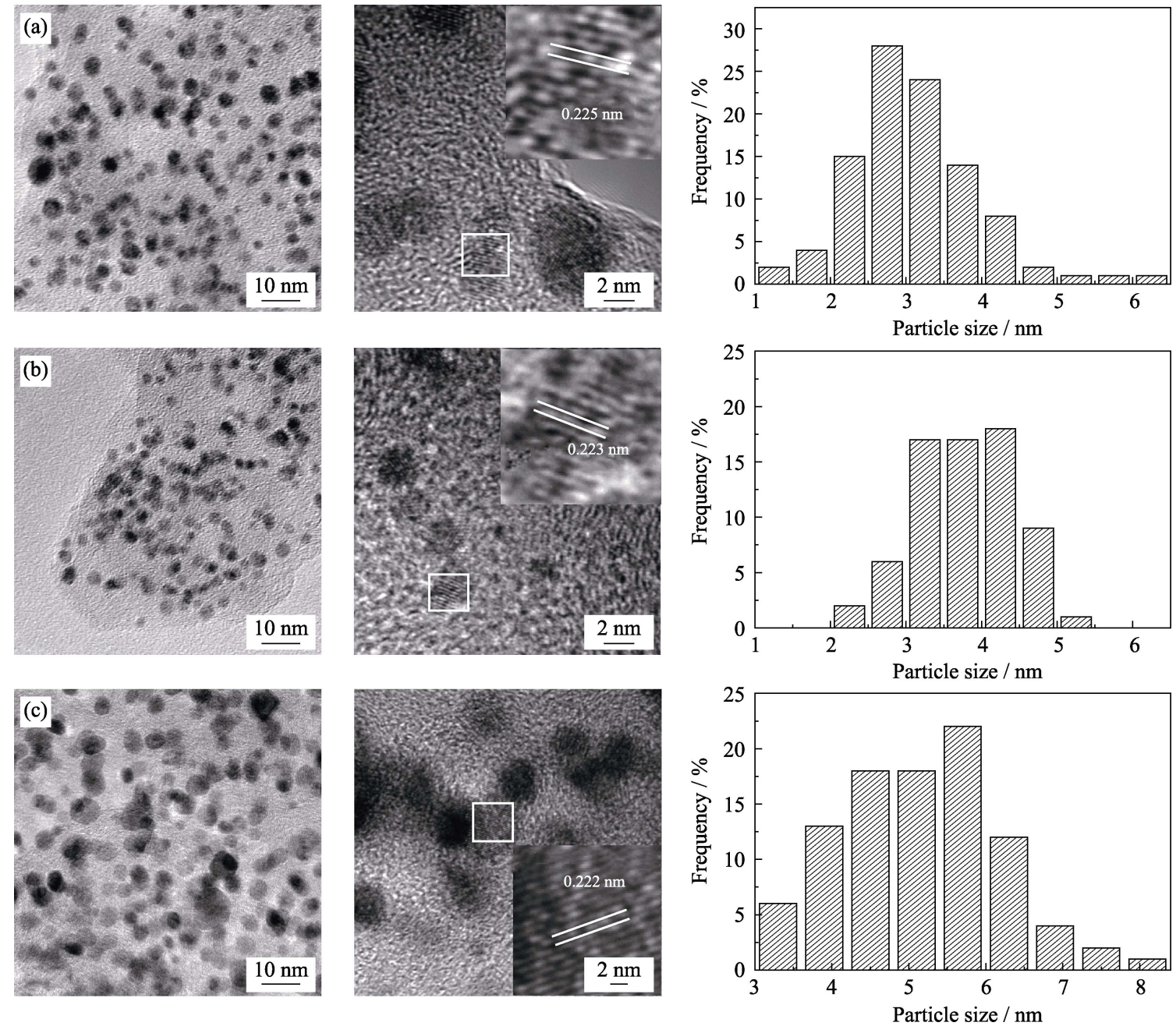

图 $2 \mathrm{Pt}_{2.8} \mathrm{Co} / \mathrm{C}(\mathrm{a}) 、 \mathrm{Pt}_{2.8} \mathrm{Co} / \mathrm{C}-500(\mathrm{~b})$ 和 $\mathrm{TKK}-\mathrm{PtCo} / \mathrm{C}(\mathrm{c})$ 的 $\mathrm{TEM}$ 照片及粒径分布图

Fig. 2 TEM images and particle size distributions of $\mathrm{Pt}_{2.8} \mathrm{Co} / \mathrm{C}(\mathrm{a}), \mathrm{Pt}_{2.8} \mathrm{Co} / \mathrm{C}-500(\mathrm{~b})$ and $\mathrm{TKK}-\mathrm{PtCo} / \mathrm{C}(\mathrm{c})$
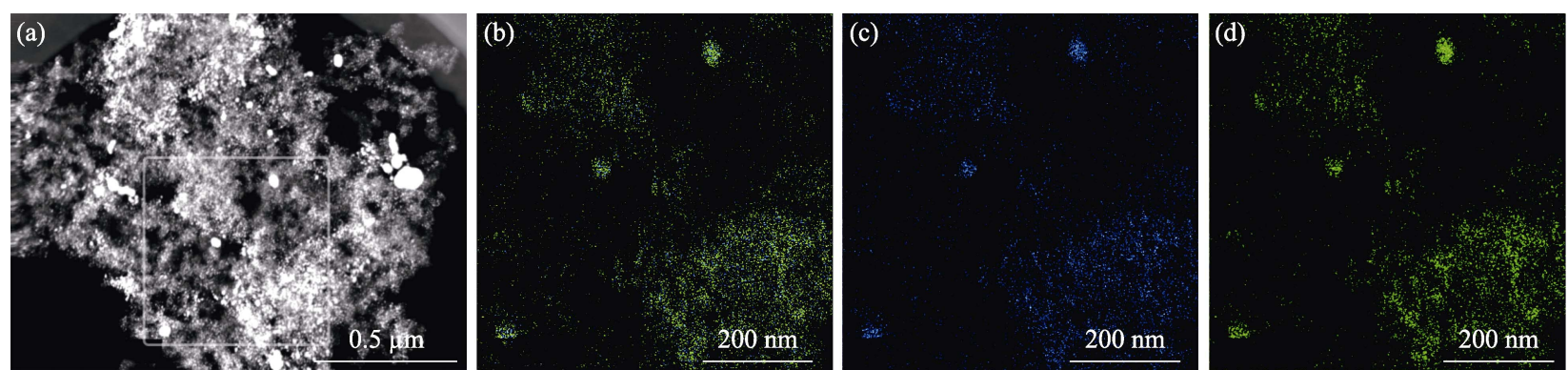

图 $3 \mathrm{Pt}_{2.8} \mathrm{Co} / \mathrm{C}-500$ 的 $\mathrm{EDS}$ 图谱

Fig. 3 EDS mappings of $\mathrm{Pt}_{2.8} \mathrm{Co} / \mathrm{C}-500$

(a) TEM image; (b) PtCo alloy; (c) Co; (d) Pt

ORR 能力。根据 Yang 等 ${ }^{[22]}$ 研究, 在前驱液中添加 三乙胺有助于提升 ORR 能力。故实验探究了三乙胺 添加量对于催化剂电化学性能的影响。

图 4(b)为添加不同三乙胺制备的 DTL- $\mathrm{Pt}_{2.8} \mathrm{Co} / \mathrm{C}$ 催化剂的 $\mathrm{MA}$ 。当加入三乙胺量为 $90 \sim 110 \mu \mathrm{L}$ 时, 所制备的 $\mathrm{DTL}-\mathrm{Pt}_{2.8} \mathrm{Co} / \mathrm{C}$ 的 $\mathrm{MA}$ 有明显提升。如 图 S3 所示, 当三乙胺的添加量为 $100 \mu \mathrm{L}$ 时,
$\mathrm{DTL}-\mathrm{Pt}_{2.8} \mathrm{Co} / \mathrm{C}$ 催化剂的半波电位比 $\mathrm{TKK}-\mathrm{PtCo} / \mathrm{C}$ 合金催化剂更高，通过 $\mathrm{LS} \mathrm{V}$ 曲线计算得到 DTL-Pt ${ }_{2.8} \mathrm{Co} / \mathrm{C}$ 的 $\mathrm{MA}$ 达到 $54.2 \mathrm{~mA} / \mathrm{mg}_{\mathrm{Pt}}$, 高于 $\mathrm{TKK}-\mathrm{PtCo} / \mathrm{C}\left(44.3 \mathrm{~mA} / \mathrm{mg}_{\mathrm{Pt}}\right)$ 。加入适量的三乙胺可 以提升催化剂的 ORR 能力, 与 Yang 等 ${ }^{[22]}$ 的研究结论 相符合。三乙胺可与酸进行反应, 如与氯化氢 $(\mathrm{HCl})$ 结合成三乙胺盐酸盐 ${ }^{[23]}$ 。在前驱液中, 三乙胺与氯 

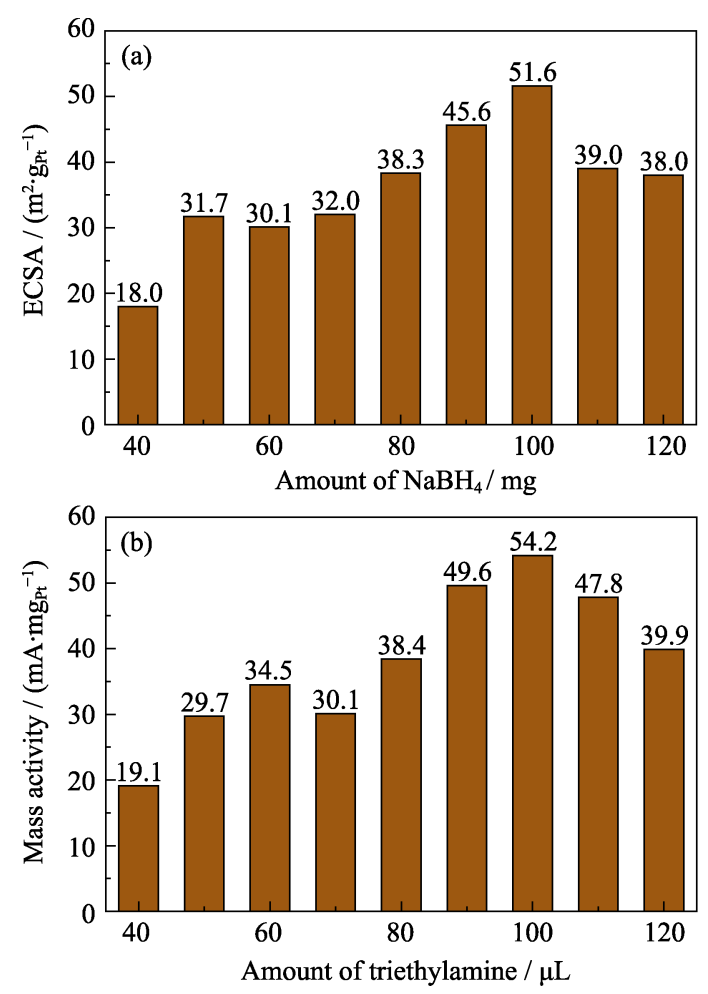

图 4 不同 $\mathrm{NaBH}_{4}$ 添加量所制备的 $\mathrm{DN}-\mathrm{Pt}_{2.8} \mathrm{Co} / \mathrm{C}$ 的 ECSA 直方图(a); 不同三乙胺添加量所制备 $\mathrm{DTL}-\mathrm{Pt}_{2.8} \mathrm{Co} / \mathrm{C}$ 的 $\mathrm{MA}$ 直方图(b)

Fig. 4 ECSA histogram for $\mathrm{DN}-\mathrm{Pt}_{2.8} \mathrm{Co} / \mathrm{C}$ with different amounts of $\mathrm{NaBH}_{4}$ (a); MA histogram for DTL-Pt $2.8 \mathrm{Co} / \mathrm{C}$ with different amounts of triethylamine (b)

铂酸反应生成有机复合络合物，能均匀地分布在碳 基上，形成浑浊悬浮的胶体溶液，有助于金属颗粒 均一地负载在碳黑上, 从而极大影响金属颗粒的生
长过程，提升金属颗粒的电催化性能 ${ }^{[24]}$ 。

高温热处理对 $\mathrm{Pt}_{2.8} \mathrm{Co} / \mathrm{C}$ 的 $\mathrm{ECSA}$ 的影响如 图 S4(a)所示。高温热处理后, $\mathrm{Pt}_{2.8} \mathrm{Co} / \mathrm{C}$ 的 $\mathrm{ECSA}$ 均 有所下降，这是因为高温使催化剂的金属颗粒尺寸 变大, 比表面积下降，催化剂的活性位点变少。当温 度较高时, $\mathrm{Pt}_{2.8} \mathrm{Co} / \mathrm{C}$ 催化剂会出现烧结的现象, 使 催化剂金属颗粒出现较大团聚，催化剂的电化学活 性急剧下降 ${ }^{[25]}$ 。高温热处理对 $\mathrm{Pt}_{2.8} \mathrm{Co} / \mathrm{C}$ 的 $\mathrm{MA}$ 的影 响如图 $\mathrm{S} 4(\mathrm{~b})$ 所示, 高温热处理后, $\mathrm{Pt}_{2.8} \mathrm{Co} / \mathrm{C}-T$ 的 MA 都有明显提升, 这是由于高温热处理后, 催化 剂金属颗粒变成有序结构, Pt 原子和 Co 原子的有序 配位作用及有序几何作用大大提升了 ORR 能力 ${ }^{[26]}$ 。 同时 $500{ }^{\circ} \mathrm{C}$ 为最佳热处理温度, 此温度下 $\mathrm{Pt}_{2.8} \mathrm{Co} / \mathrm{C}-500$ 既无大面积的金属团聚，具有较多的 电化学活性位点, 又拥有最高的 ORR 性能。通过 $\mathrm{CV}$ 曲线(图 5(a)) 的对比得出 $\mathrm{Pt}_{2.8} \mathrm{Co} / \mathrm{C}-500$ 的氢脱附 区域面积比 $\mathrm{Pt}_{2.8} \mathrm{Co} / \mathrm{C}$ 更小, 说明 $\mathrm{Pt}_{2.8} \mathrm{Co} / \mathrm{C}-500$ 催化 剂吸附电荷能力比 $\mathrm{Pt}_{2.8} \mathrm{Co} / \mathrm{C}$ 弱。如表 1 所示, 虽然 $\mathrm{Pt}_{2.8} \mathrm{Co} / \mathrm{C}-500$ 的 ECSA 比 $\mathrm{Pt}_{2.8} \mathrm{Co} / \mathrm{C}$ 低, 但仍高于 $\mathrm{TKK}-\mathrm{PtCo} / \mathrm{C}$ 。如图 5(b)所示, 通过 LSV 曲线对比可 知, $\mathrm{Pt}_{2.8} \mathrm{Co} / \mathrm{C}-500$ 的半波电位明显高于 $\mathrm{Pt}_{2.8} \mathrm{Co} / \mathrm{C}$ 和 $\mathrm{TKK}-\mathrm{PtCo} / \mathrm{C}$ 。如表 1 所示, 其 $\mathrm{MA}$ 为 $133 \mathrm{~mA} / \mathrm{mg}_{\mathrm{Pt}}$, 是 $\mathrm{TKK}-\mathrm{PtCo} / \mathrm{C}$ 的 3 倍, 结合 $\mathrm{XRD}$ 图谱(图 1)和 TEM 照片(图 2), 说明更优的结晶度、更高的合金化程度 可有效提升催化剂的 ORR 能力。

$\mathrm{Pt}_{2.8} \mathrm{Co} / \mathrm{C}-500$ 加速稳定性测试前后的 $\mathrm{CV}$ 及 LSV 曲线如图 5(c,d)所示。由图 5(c) 可得, 经过 5000
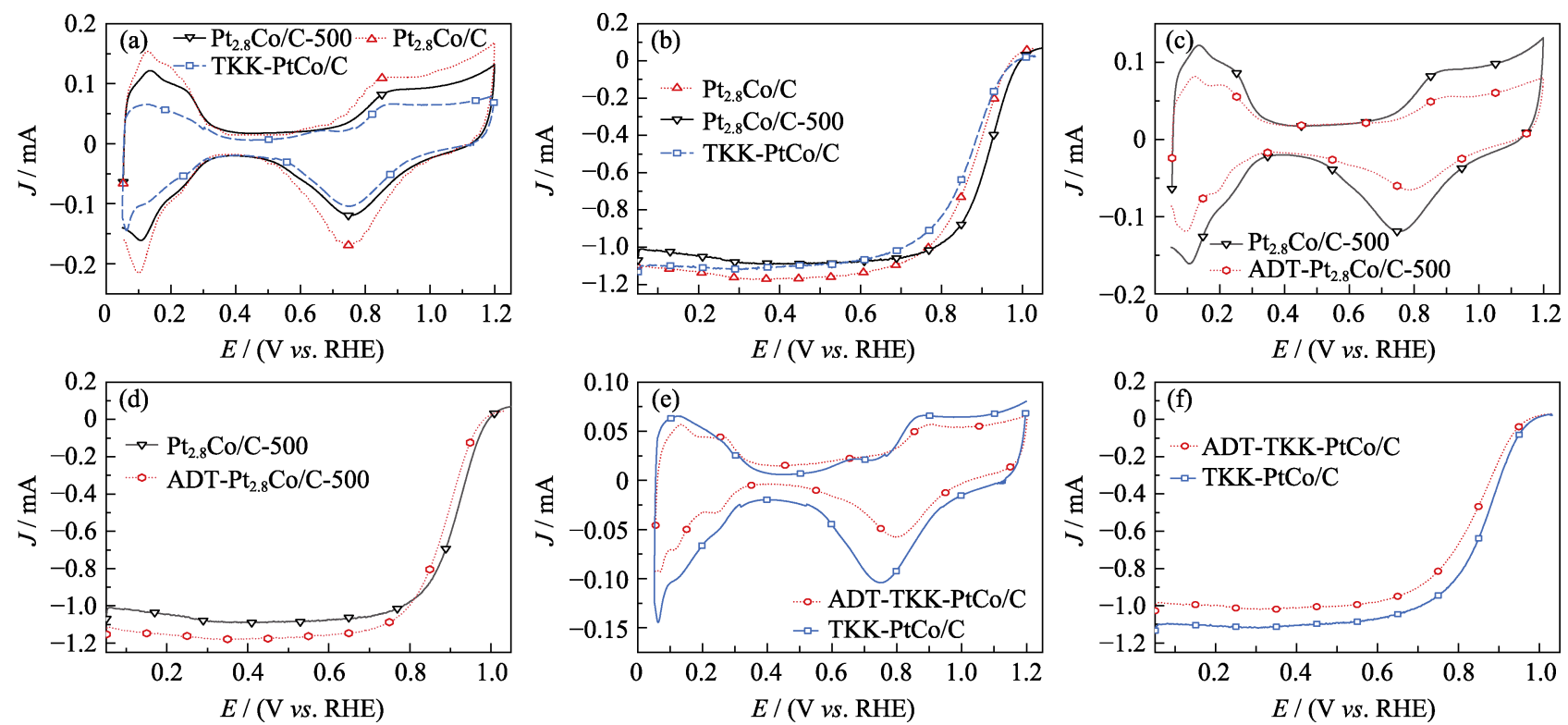

图 $5 \mathrm{Pt}_{2.8} \mathrm{Co} / \mathrm{C} 、 \mathrm{Pt}_{2.8} \mathrm{Co} / \mathrm{C}-500$ 、 $\mathrm{TKK}-\mathrm{PtCo} / \mathrm{C}$ 的 $\mathrm{CV}$ (a) 和 $\mathrm{LSV}$ 曲线(b); 加速稳定性测试(ADT)前后 $\mathrm{Pt}_{2.8} \mathrm{Co} / \mathrm{C}-500(\mathrm{c}, \mathrm{d}), \mathrm{TKK}-\mathrm{PtCo} / \mathrm{C}(\mathrm{e}, \mathrm{f})$ 的 $\mathrm{CV}(\mathrm{c}, \mathrm{e})$ 和 $\operatorname{LSV}(\mathrm{d}, \mathrm{f})$ 曲线

Fig. $5 \mathrm{CV}(\mathrm{a})$ and LSV (b) curves for $\mathrm{Pt}_{2.8} \mathrm{Co} / \mathrm{C}, \mathrm{Pt}_{2.8} \mathrm{Co} / \mathrm{C}-500$ and TKK-PtCo/C; $\mathrm{CV}(\mathrm{c}, \mathrm{e})$ and LSV(d, f) curves of $\mathrm{Pt}_{2.8} \mathrm{Co} / \mathrm{C}-500(\mathrm{c}, \mathrm{d})$ and $\mathrm{TKK}-\mathrm{PtCo} / \mathrm{C}(\mathrm{e}, \mathrm{f})$ before and after durability tests 
表 1 PtCo 合金电催化剂的 ECSA 和 MA 数据

Table 1 ECSA and MA parameters of platinum-cobalt alloy nano catalysts

\begin{tabular}{|c|c|c|}
\hline Sample & $\mathrm{ECSA} /\left(\mathrm{m}^{2} \cdot \mathrm{g}_{\mathrm{Pt}}{ }^{-1}\right)$ & $\mathrm{MA} /\left(\mathrm{mA} \cdot \mathrm{mg}_{\mathrm{Pt}}^{-1}\right)$ \\
\hline $\mathrm{Pt}_{2.8} \mathrm{Co} / \mathrm{C}$ & 51.6 & 54.2 \\
\hline $\mathrm{Pt}_{2.8} \mathrm{Co} / \mathrm{C}-500$ & 43.5 & 133.0 \\
\hline TKK-PtCo/C & 28.1 & 44.3 \\
\hline $\mathrm{ADT}-\mathrm{Pt}_{2.8} \mathrm{Co} / \mathrm{C}-500$ & 24.6 & 71.0 \\
\hline ADT-TKK-PtCo/C & 15.7 & 27.2 \\
\hline
\end{tabular}

圈的 $\mathrm{CV}$ 循环扫描后, $\mathrm{Pt}_{2.8} \mathrm{Co} / \mathrm{C}-500$ 的氢脱附区面积 减小, 这说明催化剂表面的活性位点减少, 通过 CV 曲线计算得出, $\mathrm{Pt}_{2.8} \mathrm{Co} / \mathrm{C}-500$ 的 $\mathrm{ECSA}$ (表 1) 由 $43.5 \mathrm{~m}^{2} / \mathrm{g}_{\mathrm{Pt}}$ 降到 $24.6 \mathrm{~m}^{2} / \mathrm{g}_{\mathrm{Pt}}$, 降幅 $39.4 \%$ 。由图 $5(\mathrm{~d})$ 可知, 经过 5000 圈 $\mathrm{CV}$ 循环扫描后, $\mathrm{Pt}_{2.8} \mathrm{Co} / \mathrm{C}-500$ 的半波电位明显减小, 负偏移 $34 \mathrm{mV}$, 如表 1 所示, $\mathrm{MA}$ 由 $133 \mathrm{~mA} / \mathrm{mg}_{\mathrm{Pt}}$ 降低到 $71 \mathrm{~mA} / \mathrm{mg}_{\mathrm{Pt}}$, 降幅为 $46 \%$ 。 TKK-PtCo/C 加速稳定性测试的 CV 及 LSV 曲线如图 5(e,f)所示, 经过 5000 圈 CV 循环扫描后, $\mathrm{TKK}-\mathrm{PtCo} / \mathrm{C}$ 的 ECSA 和 MA 都明显降低, 其 ECSA 由 $28.1 \mathrm{~m}^{2} / \mathrm{g}_{\mathrm{Pt}}$ 降到 $15.7 \mathrm{~m}^{2} / \mathrm{g}_{\mathrm{Pt}}$ (表 1 ), 降幅为 $27.5 \%$ 。 如图 5(f)所示, 经过加速稳定性测试后, TKK-PtCo/C 的半波电位负偏移 $23 \mathrm{mV}, \mathrm{MA}$ 由 $44.3 \mathrm{~mA} / \mathrm{mg}_{\mathrm{Pt}}$ 减小 到 $27.2 \mathrm{~mA} / \mathrm{mg}_{\mathrm{Pt}}$ (表 1), 降幅为 $38.6 \%$ 。这说明 $\mathrm{Pt}_{2.8} \mathrm{Co} / \mathrm{C}-500$ 的初始电化学性能虽然较高, 但其稳 定性比 $\mathrm{TKK}-\mathrm{PtCo} / \mathrm{C}$ 弱。这是由于在酸性条件下, 催 化剂经过加速稳定性测试后, 其金属表面的原始结 构被破坏, 部分金属颗粒脱落 ${ }^{[27-28]}$, 导致催化剂电 化学性能降低。如图 S5 所示, 加速稳定性测试后, $\mathrm{Pt}_{2.8} \mathrm{Co} / \mathrm{C}-500$ 电催化剂金属颗粒出现明显团聚, 其 平均颗粒尺寸由 $3.85 \mathrm{~nm}$ 增加到 $5.35 \mathrm{~nm}$, 晶格间距 由 $0.223 \mathrm{~nm}$ 增加到 $0.233 \mathrm{~nm}$, 这论证了其原始结构 被破坏是导致电化学性能衰减的主要原因之一。此 外, 碳载体结构受到电化学腐蚀的影响, 也会导致 催化剂的活性位点减少、氧还原反应能力减弱 ${ }^{[8]}$ 。

\section{3 结论}

本研究通过实验优化后确定：添加 $100 \mathrm{mg}$ 硼氢 化钠及 $100 \mu \mathrm{L}$ 三乙胺在 $500{ }^{\circ} \mathrm{C}$ 高温热处理的条件 下制备的 $\mathrm{Pt}_{2.8} \mathrm{Co} / \mathrm{C}-500$ 纳米合金电催化剂的电化学 性能最优。与商用 $\mathrm{TKK}-\mathrm{PtCo} / \mathrm{C}$ 电催化剂相比, $\mathrm{Pt}_{2.8} \mathrm{Co} / \mathrm{C}-500$ 合金纳米电催化剂分散较好、合金颗 粒尺寸更小且电化学活性面积更大, 催化剂活性位 点更多, $\mathrm{Pt}_{2.8} \mathrm{Co} / \mathrm{C}-500$ 的 ECSA 达到 $43.5 \mathrm{~m}^{2} / \mathrm{g}_{\mathrm{Pt}}$, 超 过商用 $\mathrm{TKK}-\mathrm{PtCo} / \mathrm{C}$ 电催化剂的 $28.1 \mathrm{~m}^{2} / \mathrm{g}_{\mathrm{Pt}}$; 特别在 氧还原反应性能上, $\mathrm{Pt}_{2.8} \mathrm{Co} / \mathrm{C}-500$ 的质量活性达到
$133 \mathrm{~mA} / \mathrm{mg}_{\mathrm{Pt}}$, 是商用 $\mathrm{TKK}-\mathrm{PtCo} / \mathrm{C}$ 电催化剂的 3 倍, 拥有更强的 ORR 能力。经过 5000 圈 $\mathrm{CV}$ 扫描后, $\mathrm{TKK}-\mathrm{PtCo} / \mathrm{C}$ 的稳定性较优, $\mathrm{Pt}_{2.8} \mathrm{Co} / \mathrm{C}-500$ 的稳定性 需进一步加强。本研究合成的 $\mathrm{Pt}_{2.8} \mathrm{Co} / \mathrm{C}-500$ 可以提 升 $\mathrm{Pt}$ 的利用率, 其更高的质量活性可减少催化剂使 用量，降低质子交换膜燃料电池的成本。

\section{补充材料}

本文相关补充材料可登陆 https://doi.org/10.15541/ jim20200253 查看。

\section{参考文献:}

[1] 毛宗强. 燃料电池. 化学工业出版社, 2005.

[2] 孙世刚等. 电催化. 北京: 化学工业出版社, 2013: 663 .

[3] SUI S, WANG X, ZHOU X, et al. A comprehensive review of Pt electrocatalysts for the oxygen reduction reaction: nanostructure, activity, mechanism and carbon support in PEM fuel cells. $J$. Mater. Chem. A, 2017, 5(5): 1808-1825.

[4] ZHAO L, WANG Q, ZHANG X, et al. Combined electron and structure manipulation on Fe-containing $\mathrm{N}$-doped carbon nanotubes to boost bifunctional oxygen electrocatalysis. ACS Appl. Mater. Int., 2018, 10(42): 35888-35895.

[5] GAN J, ZHANG J, ZHANG B, et al. Active sites engineering of $\mathrm{Pt} / \mathrm{CNT}$ oxygen reduction catalysts by atomic layer deposition. $J$. Energy Chem., 2020, 45: 59-66.

[6] JUNG W S. Study on durability of Pt supported on graphitized carbon under simulated start-up/shut-down conditions for polymer electrolyte membrane fuel cells. J. Energy Chem., 2018, 27(1): 326-334.

[7] PENG H, MO Z, LIAO S, et al. High performance Fe- and Ndoped carbon catalyst with graphene structure for oxygen reduction. Sci. Rep.-UK, 2013, 3(1): 1765.

[8] LUO Y, FENG J Z, FENG J, et al. Research progress on advanced carbon materials as Pt support for proton exchange membrane fuel cells. J. Inorg. Mater., 2020, 35(4): 407-415.

[9] LIU J, HE T, WANG Q, et al. Confining ultrasmall bimetal alloys in porous $\mathrm{N}$-carbon as scalable and sustainable electrocatalysts for rechargeable Zn-air batteries. J. Mater. Chem. A, 2019, 7: 12451.

[10] LOUISIA S, THOMAS Y R J, LECANTE P, et al. Alloyed $\mathrm{Pt}_{3} \mathrm{M}$ $(\mathrm{M}=\mathrm{Co}, \mathrm{Ni})$ nanoparticles supported on $\mathrm{S}-$ and $\mathrm{N}$-doped carbon nanotubes for the oxygen reduction reaction. Beilstein J. Nanotech., 2019, 10(1): 1251-1269.

[11] XU W, LYU F, BAI Y, et al. Porous cobalt oxide nanoplates enriched with oxygen vacancies for oxygen evolution reaction. Nano Energy, 2018, 43: 110-116.

[12] CHEN Z, WANG Q, ZHANG X, et al. N-doped defective carbon with trace Co for efficient rechargeable liquid electrolyte-/all-solid-state Zn-air batteries. Science Bulletin, 2018, 63(9): 548-555.

[13] BEARD B C. The structure and activity of Pt-Co alloys as oxygen reduction electrocatalysts. J. Electrochem. Soc., 1990, 137(11): 3368.

[14] LI S L, YUAN X X, KONG H C, et al. Fe-PPy-TsOH/C as cathode catalyst for proton exchange membrane fuel cells. J. Inorg. Mater., 2017, 32(4): 393.

[15] BAI P, TIAN F, WANG H, et al. Electrocatalytic enhancement of 0D/1D/2D multidimensional PtCo alloy@cobalt benzoate/graphene composite catalyst for alcohol electro-oxidation. Advanced Materials 
Interfaces, 2019, 6(19): 1900946.

[16] 杨德隆, 顾军, 刘晓梦, 等. 铂基催化剂氧还原测试技术的研究 进展. 电源技术, 2019, 43(12): 2040-2043.

[17] WANG G, HILGERT J, RICHTER F H, et al. Platinum-cobalt bimetallic nanoparticles in hollow carbon nanospheres for hydrogenolysis of 5-hydroxymethylfurfural. Nat. Mater., 2014, 13(3): 293-300.

[18] ZHANG L, HAN L, LIU H, et al. Potential-cycling synthesis of single platinum atoms for efficient hydrogen evolution in neutral media. Angewandte Chemie International Edition, 2017, 56(44): 13694-13698.

[19] LIN L, ZHOU W, GAO R, et al. Low-temperature hydrogen production from water and methanol using Pt/alpha-MoC catalysts. Nature, 2017, 544(7648): 80-83.

[20] HUANG H, LI K, CHEN Z, et al. Achieving remarkable activity and durability toward oxygen reduction reaction based on ultrathin Rh-doped Pt nanowires. J. Am. Chem. Soc., 2017, 139(24): 8152-8159.

[21] STRASSER P, KOH S, ANNIYEV T, et al. Lattice-strain control of the activity in dealloyed core-shell fuel cell catalysts. Nat. Chem., 2010, 2(6): 454-460.

[22] YANG D, GU J, LIU X, et al. Monodispersed $\mathrm{Pt}_{3} \mathrm{Ni}$ nanoparticles as a highly efficient electrocatalyst for PEMFCs. Catalysts, 2019,
9(7): 588 .

[23] PAQUETTE. Encyclopedia of Reagents for Organic Synthesis. Chichester: Wiley, 1995: 8.

[24] YAO S, FENG L, ZHAO X, et al. Pt/C catalysts with narrow size distribution prepared by colloidal-precipitation method for methanol electrooxidation. J. Power Sources, 2012, 217: 280-286.

[25] ANTOLINI E. Structural parameters of supported fuel cell catalysts: the effect of particle size, inter-particle distance and metal loading on catalytic activity and fuel cell performance. Applied Catalysis B: Environmental, 2016, 181: 298-313.

[26] SAHIN N E, NAPPORN T W, DUBAU L, et al. Temperaturedependence of oxygen reduction activity on $\mathrm{Pt} / \mathrm{C}$ and $\mathrm{PtCr} / \mathrm{C}$ electrocatalysts synthesized from microwave-heated diethylene glycol method. Applied Catalysis B: Environmental, 2017, 203: $72-84$.

[27] ARUMUGAM B, TAMAKI T, YAMAGUCHI T. Beneficial role of copper in the enhancement of durability of ordered intermetallic $\mathrm{PtFeCu}$ catalyst for electrocatalytic oxygen reduction. ACS Appl. Mater. Int., 2015, 7(30): 16311-16321.

[28] KUROKI H, TAMAKI T, MATSUMOTO M, et al. Platinumiron-nickel trimetallic catalyst with superlattice structure for enhanced oxygen reduction activity and durability. Ind. Eng. Chem. Res., 2016, 55(44): 11458-11466. 


\title{
补充材料：
}

\section{铂钴合金纳米电催化剂的制备及性能研究}

\author{
朱 勇 ${ }^{1}$, 顾 军 ${ }^{1,2}$, 于 涛 ${ }^{1,3}$, 何海佟 ${ }^{1}$, 姚 点 $^{1}$
}

(1. 南京大学 物理学院, 南京 $210093 ; 2$. 南京东炎氢能源科技有限公司, 南京 211100 ; 3. 南京大学 固体微结构 物理国家重点实验室, 南京 210093)

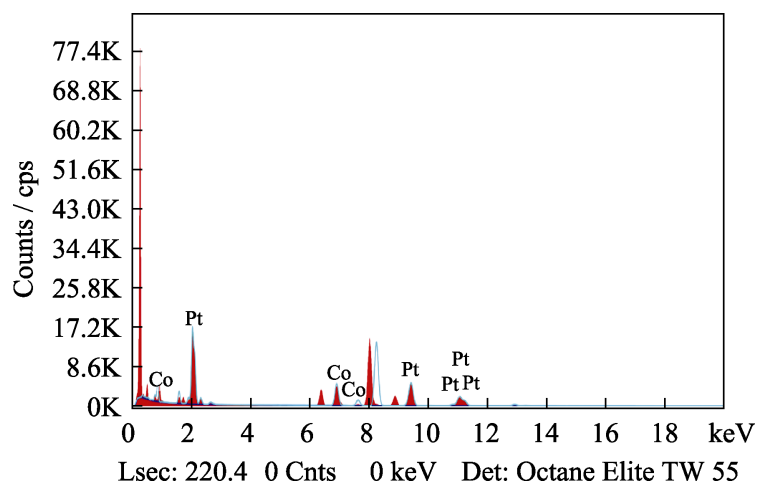

图 $\mathrm{S} 1 \quad \mathrm{Pt}_{2.8} \mathrm{Co} / \mathrm{C}-500$ 的能谱图

Fig. S1 Energy spectrum of $\mathrm{Pt}_{2.8} \mathrm{Co} / \mathrm{C}-500$
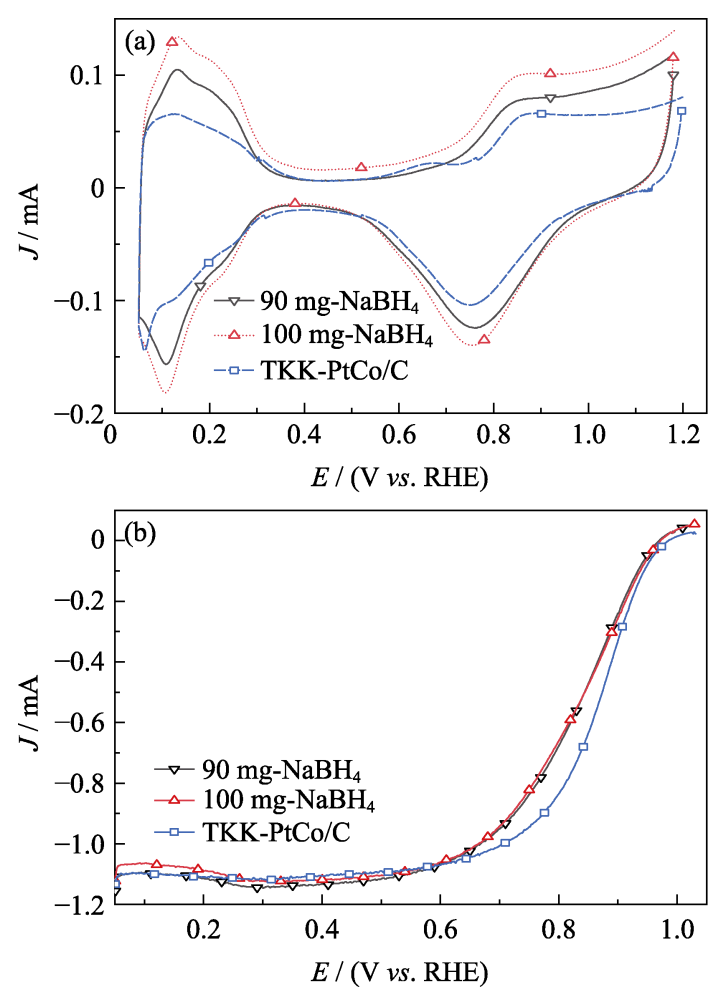

图 S2 不同 $\mathrm{NaBH}_{4}$ 添加量所制备的 $\mathrm{DN}_{-} \mathrm{Pt}_{2.8} \mathrm{Co} / \mathrm{C}$ 和 $\mathrm{TKK}-\mathrm{PtCo} / \mathrm{C}$ 合金催化剂的 CV(a)和 LSV(b)曲线

Fig. S2 CV(a) and LSV(b) curves of TKK-PtCo/C and $\mathrm{DN}-\mathrm{Pt}_{2.8} \mathrm{Co} / \mathrm{C}$

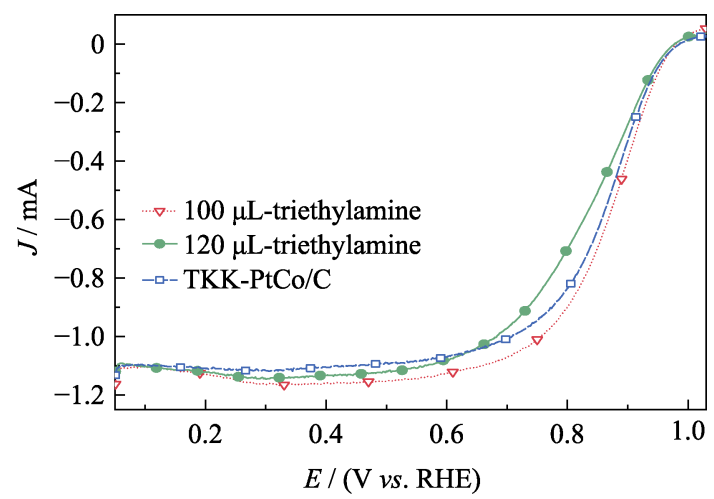

图 S3 不同三乙胺添加量所制备的 $\mathrm{DTL}-\mathrm{Pt}_{2.8} \mathrm{Co} / \mathrm{C}$ 的 LSV 曲线

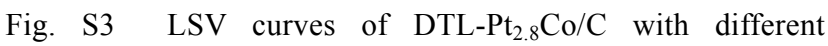
triethylamine
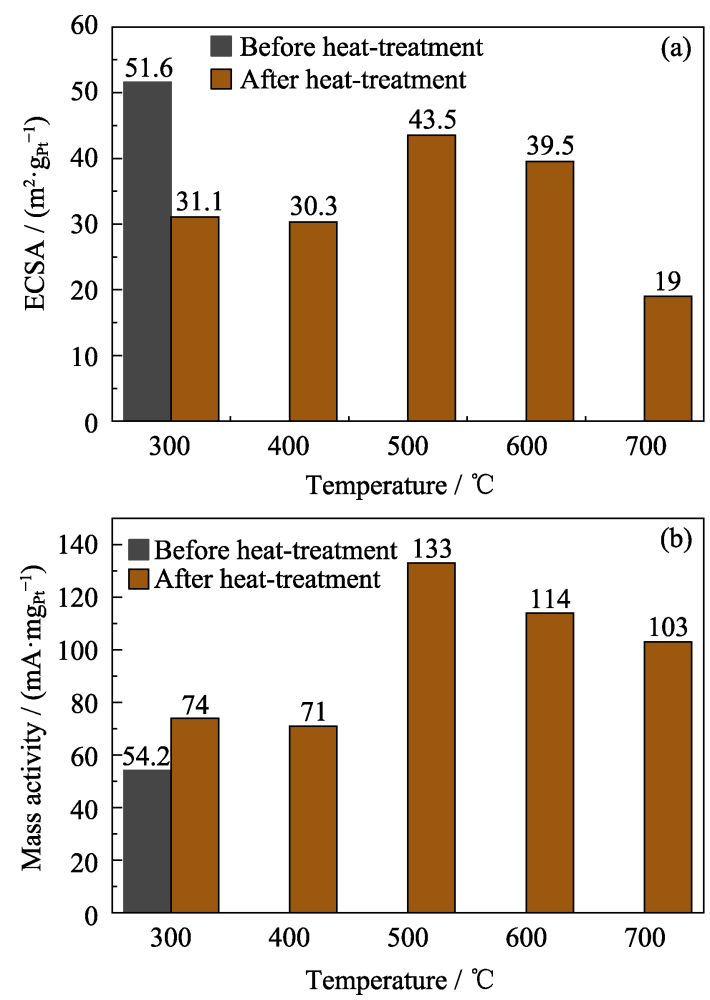

图 S4 高温热处理前后的 $\mathrm{Pt}_{2.8} \mathrm{Co} / \mathrm{C}$ 电化学活性面积(a)和质 量活性(b)直方图

Fig. S4 ECSA (a) and MA (b) histograms of $\mathrm{Pt}_{2.8} \mathrm{Co} / \mathrm{C}$ with different heat-treatment temperatures 

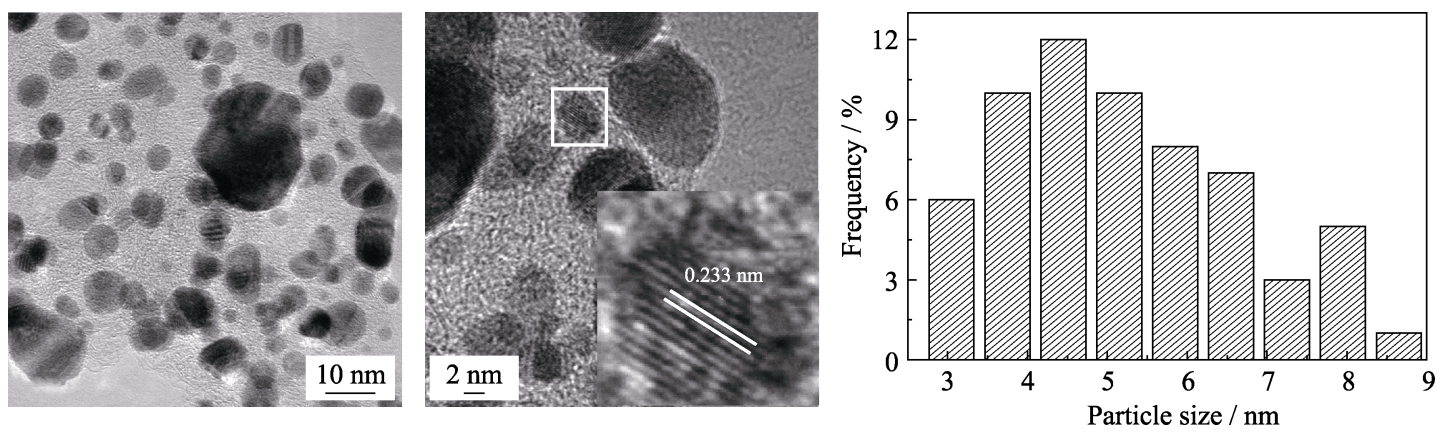

图 S5 ADT-Pt ${ }_{2.8} \mathrm{Co} / \mathrm{C}-500$ 的 TEM 照片及粒径分布图

Fig. S5 TEM images and particle size distribution of ADT-Pt ${ }_{2.8} \mathrm{Co} / \mathrm{C}-500$ 